\section{Light Microscopic Detection of} Mansonella ozzardi Parasitemias

To the Editor-Under the heading "An incidental finding from a blood smear," a case report in the Photo Quiz section of Clinical Infectious Diseases appears to show blood smears of Mansonella ozzardi microfilariae $[1,2]$. With this letter, we wish to clarify for readers that M. ozzardi microfilariae are typically about $160-200 \mu \mathrm{m}$ in length [3] and are thus not shown at $\times 100$ magnification as in Hidron et al [1,2]. Furthermore, from a clinical training perspective, it is also important to point out that while a powerful microscope objective lens $(\mathrm{eg}, \times 100)$ can be used to help identify microfilariae, they are not easily found with them. This counterintuitive fact, that filarial parasitemias are often missed with high-magnification objectives (eg, $\times 100)$, was actually used by Sir Patrick Manson (the founder of the London School of Hygiene and Tropical Medicine) to advocate the opening of specialist tropical medicine training institutions before they existed:

"Ten chances to one if one asks a student, or even a medical practitioner, to set about examining a patient for filariae he will prepare a very fine film of blood, such as would be suitable for the demonstration of bacteria, and that he would set to work to examine it with a twelfth of an inch immersion lens.... Although there may be tens of millions of filariae in the patient's blood the chances are they will not be discovered by such means.... Most people think that when they have to make a microscopic examination the more microscope they have the better. As a rule, the reverse is truth. Filariae should be sought with an inch objective otherwise they will be missed." [4]

Given that M. ozzardi microfilaremias are still often detected and diagnosed using the same light microscopy-based techniques that Manson used 100 years ago $[2,3]$, his advice on the matter is still beneficial for clinical diagnostic training today. It is, thus, important (in our view) that readers of this journal who are interested in trying to diagnose $M$. ozzardi microfilaremias are made aware that they are most easily found with a $\times 10$ objective $[5,6]$. It is also important to point out that the greatest challenge for accurately diagnosing Colombian M. ozzardi microfilaremias is discriminating them from Mansonella perstans microfilaremias, which are not effectively treated with ivermectin [3], and that it is not always easy to see that the tails of M. ozzardi microfilariae are "devoid of nuclei" (see Figure $1 B$ in [1]). We recommend polymerase chain reaction (PCR)-based diagnostic techniques for definitive filarial parasite identifications, specifically the assay of Tang et al [7]. This ribosomal DNA-targeting assay can detect and discriminate all filarial parasites that commonly cause parasitemias in humans and is also more sensitive than light microscopy-based diagnosis $[3,8]$. Other mitochondrial DNA-targeting PCR-based assays have also been successfully used for this purpose [9], although it is worth noting that recent research has shown that these PCR-based techniques (including the popular $\mathrm{CO} 1$ barcoding technique) can be unreliable for M. ozzardi diagnosis and arguably should thus be avoided [10].

\section{Note}

Potential conflicts of interest. Both authors: No reported conflicts. Both authors have submitted the ICMJE Form for Disclosure of Potential Conflicts of Interest. Conflicts that the editors consider relevant to the content of the manuscript have been disclosed.

James Lee Crainey ${ }^{\circledR}$ and Sérgio Luiz Bessa Luz

Laboratório de Ecologia de Doenças Transmissíveis na Amazônia, Instituto Leônidas e Maria Deane/Instituto Leônidas e Maria Deane/Fundação Oswaldo Cruz, Manaus, Brazil

\section{References}

1. Hidron A, Gálvez K, Madrid C, et al. Photo quiz (question). An incidental finding from a blood smear. Clin Infect Dis 2012; 55:106.
2. Hidron A, Gálvez K, Madrid C, et al. Photo quiz (answer). An incidental finding from a blood smear. Clin Infect Dis 2012; 55:155-6.

3. Ta-Tang TH, Crainey JL, Post RJ, Luz SL, Rubio JM. Mansonellosis: current perspectives. Res Rep Trop Med 2018; 9:9-24.

4. Manson P. An introductory address: on the necessity for special education in tropical medicine. Lancet 1897; 150:842-5.

5. World Health Organization. Bench aids for the diagnosis of filarial infections. 1997. Available at: http://apps.who.int/iris/bitstream/ handle/10665/37156/9241544899_eng.pdf;jsessionid $=4$ CC86FC5F4D 98C35BE0827F102EC$6 \mathrm{CC} 0$ ? sequence $=1$. Accessed 6 October 2018.

6. World Health Organization. Monitoring and epidemiological assessment of the programme to eliminate lymphatic filariasis at implementation unit level. 2005. Available at: http://apps.who.int/ iris/bitstream/handle/10665/69172/WHO_CDS_ CPE_CEE_2005.50.pdf? sequence $=1$ \& isAllowed $=\mathrm{y}$. Accessed 6 October 2018.

7. Tang TH, López-Vélez R, Lanza M, Shelley AJ, Rubio JM, Luz SL. Nested PCR to detect and distinguish the sympatric filarial species Onchocerca volvulus, Mansonella ozzardi and Mansonella perstans in the Amazon region. Mem Inst Oswaldo Cruz 2010; 105:823-8.

8. Medeiros JF, Almeida TA, Silva LB, et al. A field trial of a PCR-based Mansonella ozzardi diagnosis assay detects high-levels of submicroscopic M. ozzardi infections in both venous blood samples and FTA card dried blood spots. Parasit Vectors 2015; 8:280.

9. Tavares da Silva LB, Crainey JL, Ribeiro da Silva TR, et al. Molecular verification of New World Mansonella perstans parasitemias. Emerg Infect Dis 2017; 23:545-7.

10. Crainey JL, Marín MA, Silva TRRD, et al. Mansonella ozzardi mitogenome and pseudogene characterisation provides new perspectives on filarial parasite systematics and CO-1 barcoding. Sci Rep 2018; 8:6158.

Correspondence: J. L. Crainey, Instituto Leônidas e Maria Deane/ILMD/FIOCRUZ, Laboratório de Ecologia de Doenças Transmissíveis na Amazônia, 476 Rua Teresina, Adrianópolis, Manaus, Amazonas, Brazil (lee.crainey@gmail.com).

\section{Clinical Infectious Diseases ${ }^{\circledR} \quad$ 2019;68(12):2156}

(C) The Author(s) 2018. Published by Oxford University Press for the Infectious Diseases Society of America. All rights reserved. For permissions, e-mail: journals.permissions@oup.com. DOI: 10.1093/cid/ciy1055

\section{Human Immunodeficiency Virus-exposed Uninfected Infants: Surviving and Thriving or Overlooked by Success?}

Keywords. HIV exposed uninfected; HEU; HIV; maternal antiretroviral therapy; infectious morbidity.

To тHE Editor-Globally, 1.4 million women who live with human immunodeficiency virus (HIV) give birth annually, and 1.25 million of their HIV-exposed 
infants remain HIV uninfected. The public health success of peri- and post-natal HIV transmission prevention is tempered by the realization that HIV-exposed uninfected (HEU) infants and children survive and thrive less well than HIV-unexposed (HU) children in comparable settings. Across all settings, compared to HU infants, HEU infants are vulnerable to higher rates and greater severity of infectious morbidity [1-3], and in low- and middle-income countries (LMICs) specifically, they experience a $20 \%-30 \%$ increased risk for preterm birth and $80 \%-90 \%$ increased risk for mortality $[4,5]$.

Therefore, we applaud the seminal work of Goetghebuer and colleagues who established in a Belgian HEU infant cohort that initiation of maternal antiretroviral therapy (ART) before pregnancy reduced the risk of infant infection-related hospitalizations [6]. In this cohort of $132 \mathrm{HEU}$ infants and $123 \mathrm{HU}$ infants, risk for infection-related hospitalization was highest in HEU infants of mothers who initiated ART during pregnancy (adjusted hazard ration [aHR], 3.84; 95\% confidence interval [CI], 1.69-8.71). However, rates in HEU infants whose mothers were already on ART at conception (aHR, 1.42; 95\% CI, 0.583.48) were similar to those in HU infants. Furthermore, this is the first study to demonstrate an association between increased infectious morbidity risk and the well-described altered HEU infant immune phenotype $[7,8]$. Goetghebuer and colleagues found that maternal and newborn monocyte activation and reduced transfer of maternal antibodies were most pronounced when maternal ART was initiated during pregnancy as opposed to before conception. These immune alterations, which occur in utero and are measurable at birth, predicted risk for infection-related hospitalization. That maternal ART from conception appeared to normalize this altered immune phenotype and largely eliminate infectious morbidity risk in HEU infants is extremely promising.

As the authors recognize, this study was conducted in a high-income country with a low HIV prevalence where women living with HIV are not encouraged to breastfeed, and these findings cannot be generalized to eastern and southern Africa where $85 \%$ of all HEU infants are born. In LMICs, but not in high-income countries, women who live with HIV and conceive while on ART are at a $40 \%$ (meta-analysis risk ratio, 1.41; 95\% CI, 1.22-1.63) higher risk of preterm delivery than those who initiate ART during pregnancy [9]. Preterm birth places infants at substantially higher risk for morbidity and mortality during infancy [10]. Therefore, the immunological benefits for HEU infants that are derived from a mother being on ART from conception may be offset in LMICs by adverse consequences of preterm birth. This is just one example of the complexities in the pathways to HEU infant vulnerability. Although it is reasonably expected that in the universal test and treat era where improved maternal health and safer prolonged breastfeeding will benefit all HEU children, we need to provide definitive evidence of this benefit to HEU infants who comprise up to $25 \%$ of the infant population in the highest HIV-burden countries. Investment is urgently needed to support carefully designed, appropriately sized cohort studies in settings with the highest prevalence of HEU infants and children.

\section{Notes}

Financial support. A.L.S receives salary support through early career development awards from the NIH Fogarty International Center (grant number 1K43TW010683) and the International AIDS Society (grant number 2017/518-SLO).

Potential conflicts of interest. All authors: No reported conflicts. All authors have submitted the ICMJE Form for Disclosure of Potential Conflicts of Interest. Conflicts that the editors consider relevant to the content of the manuscript have been disclosed.

$$
\begin{array}{r}
\text { Amy L. Slogrove, }{ }^{1,2, \bullet} \text { Kathleen M. Powis, }{ }^{3,4,5} \text { and } \\
\text { Mark F. Cotton }
\end{array}
$$

'Department of Paediatrics and Child Health, Faculty of Medicine \& Health Sciences, Stellenbosch University, and ${ }^{2} U k w a n d a$ Centre for Rural Health, Faculty of Medicine and Health Sciences, Stellenbosch University, Worcester, South Africa; ${ }^{3}$ Department of Immunology and Infectious Diseases, Harvard T.H. Chan School of Public Health, and ${ }^{4}$ Department of Internal Medicine and Pediatrics, Massachusetts General Hospital, Boston; ${ }^{5}$ Botswana Harvard AIDS Institute Partnership, Gaborone, Botswana: and ${ }^{6}$ Division of Infectious Diseases and Family Clinical Research Unit, Department of Paediatrics and Child Health, Faculty of Medicine and Health Sciences, Stellenbosch University, Tygerberg, South Africa

\section{References}

1. Taron-Brocard C, Le Chenadec J, Faye A, et al; France REcherche Nord\&Sud Sida-HIV HepatitesEnquete Perinatale Francaise-CO1/CO11 Study Group. Increased risk of serious bacterial infections due to maternal immunosuppression in HIVexposed uninfected infants in a European country. Clin Infect Dis 2014; 59:1332-45.

2. Epalza C, Goetghebuer T, Hainaut M, et al. High incidence of invasive group B streptococcal infections in HIV-exposed uninfected infants. Pediatrics 2010; 126:e631-8.

3. Slogrove AL, Goetghebuer T, Cotton MF, Singer J, Bettinger JA. Pattern of infectious morbidity in HIV-exposed uninfected infants and children. Front Immunol 2016; 7: doi:10.3389/ fimmu.2016.00164.

4. Zash R, Jacobson DL, Diseko M, et al. Comparative safety of dolutegravir-based or efavirenz-based antiretroviral treatment started during pregnancy in Botswana: an observational study. Lancet Glob Health 2018; 6:e804-10.

5. Brennan AT, Bonawitz R, Gill CJ, et al. A meta-analysis assessing all-cause mortality in HIV-exposed uninfected compared with HIVunexposed uninfected infants and children. AIDS 2016; 30:2351-60.

6. Goetghebuer T, Smolen KK, Adler C, et al. Initiation of anti-retroviral therapy before pregnancy reduces the risk of infection-related hospitalization in HIVexposed uninfected infants born in a high-income country. Clin Infect Dis 2018; doi:10.1093/cid/ ciy673.

7. Evans C, Jones CE, Prendergast AJ. Review HIVexposed, uninfected infants: new global challenges in the era of paediatric HIV elimination. Lancet Infect Dis 2016; 3099:1-16.

8. Ruck C, Reikie BA, Marchant A, Kollmann TR. Linking susceptibility to infectious diseases to immune system abnormalities among HIV-exposed uninfected infants. Front Immunol 2016; 7: doi:10.3389/fimmun.2016.00310.

9. Uthman OA, Nachega JB, Anderson J, et al. Timing of initiation of antiretroviral therapy and adverse pregnancy outcomes: a systematic review and meta-analysis. Lancet HIV 2017; 4:e21-30.

10. Katz J, Lee AC, Kozuki N, et al; CHERG Small-forGestational-Age-Preterm Birth Working Group. 
Mortality risk in preterm and small-for-gestational-age infants in low-income and middle-income countries: a pooled country analysis. Lancet 2013; 382:417-25.

Correspondence: A. L. Slogrove, 1 Durban Street, Worcester, 6850, Western Cape, South Africa (amy@sun.ac.za).

Clinical Infectious Diseases ${ }^{\circledR} \quad$ 2019;68(12):2157-8

(C) The Author(s) 2018. Published by Oxford University Press for the Infectious Diseases Society of America. All rights reserved. For permissions, e-mail: journals.permissions@oup.com. DOI: $10.1093 /$ cid/ciy1056

\section{Reply to Slogrove et al}

We would like to thank Slogrove and colleagues for the positive comments on our manuscript and for emphasizing the need to provide definitive evidence of the benefit of controlling maternal human immunodeficiency virus (HIV) infections for the health of infants born in low- and middle-income countries (LMIC), where the burden of HIV infection is highest [1]. We agree that the pathways leading to the vulnerability of HIV-exposed, uninfected (HEU) infants may not be identical in LMIC and in high-income countries (HIC), and that the potential role of specific factors has to be determined. As proposed by Slogrove and colleagues, the decreased risk of hospitalization observed in our study for those infections associated with the initiation of antiretroviral therapy (ART) before pregnancy may be offset by an increased risk of premature delivery in women living in LMIC [2]. On the other hand, contrary to HIC, women living with HIV in LMIC are encouraged to breastfeed. Although the evidence from HIC is less consistent [3], there is strong supportive evidence for a protective effect of breastfeeding on infectious morbidity in LMIC [4]. Through a diversity of immunological components, breastfeeding could reduce the immunological risk of severe infections after birth and thereby mitigate the impact of immune alterations induced by in utero exposure to maternal HIV infection. In our study, maternal and newborn immune activation predicted the risk of hospitalization due to infection in infants born to mothers who initiated ART during pregnancy [5]. Immune activation is commonly observed in adults living in LMIC, independently of HIV infection [6]. Therefore, the potential for ART to correct immune activation in women living with HIV may be lower in LMIC as compared to HIC, and this could mitigate the impact of ART initiation before pregnancy on infants' susceptibility to infectious diseases.

Although the vulnerability of HEU infants living in different settings could involve different factors, it is essential to recognize that this vulnerability is a global public health issue. An increased susceptibility of HEU infants to severe infections is observed in both LMIC and HIC, suggesting that common determinants are playing a critical role $[7,8]$. Identifying these determinants has the potential to positively impact the health of HEU infants worldwide. To meet this challenge, researchers in HIC and LMIC should join efforts and integrate both intensive studies on relatively small study populations and larger studies that are powered to determine the impact of key environmental factors on clinical outcomes. Control of maternal HIV infection before pregnancy and progress in our understanding of the immunobiology of infant exposure to maternal HIV infection provide unprecedented opportunities to further improve the health of children born to HIV-infected mothers.

\section{Note}

Potential conflicts of interest. A. M.s institution has received fees from GlaxoSmithKline Vaccines, outside the submitted work. G. A.'s institution has received grants from Gilead Sciences and the Bill and Melinda Gates Foundation (grant numbers OPP1032817, OPP1097381, and OPP1114729). T. R. K.'s institution has received grants from the National Institute for Allergy and Infectious Diseases and the Canada Institutes for Health Research. All other authors report no potential conflicts. All authors have submitted the ICMJE Form for Disclosure of Potential Conflicts of Interest. Conflicts that the editors consider relevant to the content of the manuscript have been disclosed.

Tessa Goetghebuer, ${ }^{1}$ Kinga K. Smolen, Catherine Adler, ${ }^{1}$ Jishnu Das, ${ }^{3,4}$ Trevor McBride, ${ }^{5}$ Gaby Smits, ${ }^{6}$ Sandra Lecomte, ${ }^{2}$ Edwige Haelterman, Patricia Barlow, ${ }^{7}$ Pedro A. Piedra, ${ }^{5,6}$ Fiona van der Klis, ${ }^{8}$ Tobias R. Kollmann, ${ }^{9}$ Douglas A. Lauffenburger, ${ }^{4}$ Galit Alter, ${ }^{3}$ Jack Levy, ${ }^{1}$ and Arnaud Marchant ${ }^{2}$

'Department of Pediatrics, Hôpital Saint-Pierre, Brussels, and ${ }^{2}$ Institute for Medical Immunology, Université libre de Bruxelles, Charleroi, Belgium; ${ }^{3}$ Ragon Institute of Massachusetts General Hospital, Massachusetts Institute of Technology and Harvard University, and ${ }^{4}$ Department of Biological Engineering. Massachusetts Institute of Technology, Cambridge, Massachusetts; ${ }^{5}$ Department of Molecular Virology and Microbiology, and ${ }^{6}$ Department of

Pediatrics, Baylor College of Medicine, Houston, Texas; ${ }^{7}$ Department of Obstetrics and Gynecology, Hôpital Saint-

Pierre, Brussels, Belgium; ${ }^{8}$ National Institute of Health and the Environment, Bilthoven, The Netherlands; and ${ }^{9}$ Division of Infectious Diseases, Department of Pediatrics, University of British Columbia, Vancouver, Canada

\section{References}

1. Slogrove AL, Powis KM, Cotton MF. HIV exposed uninfected infants: surviving and thriving or overlooked by success? Clin Infect Dis. 2018 Dec 11. [Epub ahead of print]. doi:10.1093/cid/ciy1056

2. Uthman OA, Nachega JB, Anderson J, et al. Timing of initiation of antiretroviral therapy and adverse pregnancy outcomes: a systematic review and meta-analysis. Lancet HIV 2017; 4:e21-30.

3. Størdal K, Lundeby KM, Brantsæter AL, et al. Breast-feeding and infant hospitalization for infections: large cohort and sibling analysis. J Pediatr Gastroenterol Nutr 2017; 65:225-31.

4. Victora CG, Bahl R, Barros AJ, et al; Lancet Breastfeeding Series Group. Breastfeeding in the 21st century: epidemiology, mechanisms, and lifelong effect. Lancet 2016; 387:475-90.

5. Goetghebuer T, Smolen KK, Adler C, et al Initiation of anti-retroviral therapy before pregnancy reduces the risk of infection-related hospitalization in HIV-exposed uninfected infants born in a high-income country. Clin Infect Dis 2018.

6. Marchant A, Sadarangani M, Garand M, et al. Maternal immunisation: collaborating with mother nature. Lancet Infect Dis 2017; 17:e197-208

7. Slogrove AL, Goetghebuer T, Cotton MF, Singer J, Bettinger JA. Pattern of infectious morbidity in HIV-exposed uninfected infants and children. Front Immunol 2016; 7:1-8.

8. Ruck C, Reikie BA, Marchant A, Kollmann TR, Kakkar F. Linking susceptibility to infectious diseases to immune system abnormalities among HIV-exposed uninfected infants. Front Immunol 2016; 7:1-12. 